\title{
D-brane instantons on non-Spin cycles
}

\section{Per Berglund $^{a}$ and Iñaki García-Etxebarria ${ }^{b}$}

\author{
${ }^{a}$ Department of Physics, University of New Hampshire, \\ Durham, NH 03824, U.S.A. \\ ${ }^{b}$ Theory Group, Physics Department, CERN, \\ CH-1211, Geneva 23, Switzerland \\ E-mail: per.berglund@unh.edu, inaki@cern.ch
}

\begin{abstract}
We show that non-Spin cycles in type IIB Calabi-Yau orientifold compactifications with vanishing $B$ field naturally support orientifold-invariant D-brane instantons. These instantons, associated to the holomorphic cotangent bundle of the non-Spin cycle, can lead to non-perturbative corrections to the superpotential.
\end{abstract}

Keywords: D-branes, Nonperturbative Effects, Brane Dynamics in Gauge Theories, Intersecting branes models

ARXIV EPRINT: 1210.1221 


\section{Contents}

1 Introduction 1

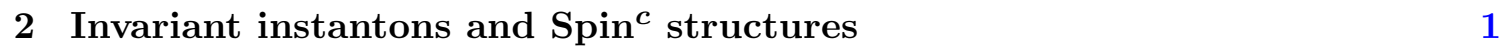

3 An invariant instanton on $\mathbb{P}^{2} \quad 2$

3.1 Neutral zero modes 2

3.2 Charged zero modes 3

4 Small volume interpretation $\quad 6$

$\begin{array}{lll}5 & \text { Conclusions } & 7\end{array}$

\section{Introduction}

Moduli stabilization is a fundamental problem when constructing realistic models of particle physics in string theory. In IIB string theory (or F-theory), the closed string moduli in the Kähler sector are stabilized by non-perturbative contributions to the superpotential. There are various promising scenarios in which non-perturbative effects, in the form of D-brane instantons, give rise to moduli stabilization, see e.g. [1, 2].

When studying contributions to the superpotential, a natural class of candidate instantons arises from D-branes wrapping rigid cycles. These cycles are often non-Spin, and in particular del Pezzo surfaces occur frequently. For non-Spin cycles one needs to consider a $\operatorname{Spin}^{c}$ structure for the worldvolume degrees of freedom instead of an ordinary Spin structure [3].

In the case of ordinary line bundles this is commonly phrased as introducing a "halfquantized bundle" on the instanton. However, this half-quantized bundle necessarily clashes with the requirement of invariance under the orientifold involution. It is well know how to restore invariance under the orientifold by introducing a half-quantized $B$-field (see for example $[4,5])$.

Nevertheless, whether there is a non-perturbative contribution in the original $B=0$ case remains an open question. In this paper we show that when $B=0$ there does exist an invariant rank 2 instanton with the right properties to contribute to the superpotential.

\section{Invariant instantons and $\operatorname{Spin}^{c}$ structures}

We will use the sheaf description of D-branes, see [6] for a good review. In our case these sheaves will be ordinary vector bundles with support on the instanton divisor. Consider the case of a surface $\mathcal{S}$ in an ambient Calabi-Yau space $X$, with inclusion map $i: \mathcal{S} \hookrightarrow X$, and 
take a sheaf $i_{*} \mathcal{E}$ describing the instanton wrapping $\mathcal{S}$. This corresponds to an instanton with bundle $\mathcal{E} \otimes \sqrt{K_{\mathcal{S}}^{V}}[6,7]$. In particular, this construction automatically gives rise to a well-defined $\operatorname{Spin}^{c}$ structure on the D-brane. A necessary condition, when $B=0$, for the instanton to be invariant under the orientifold involution is [8]:

$$
\sigma^{*}\left(\mathcal{E}^{\vee}\right) \otimes K_{\mathcal{S}}=\mathcal{E},
$$

where $\sigma$ is the orientifold involution. A solution to this equation is given by $\mathcal{E}=\Omega$, where $\Omega$ is the dual of the holomorphic tangent bundle of $\mathcal{S}$, or equivalently the sheaf of holomorphic one-forms. In the remainder of this paper we show in a particular example that the corresponding instanton has the right structure to contribute to the non-perturbative superpotential.

\section{An invariant instanton on $\mathbb{P}^{2}$}

For concreteness we focus on (the complex Calabi-Yau cone over) $\mathbb{P}^{2}$, the simplest example of a non-Spin manifold, and return to the general case in the conclusions. We parameterize $X$, our Calabi-Yau manifold, by the coordinates $(s, t, u, w)$, identified under the rescalings $(s, t, u, w) \sim\left(\lambda s, \lambda t, \lambda u, \lambda^{-3} w\right)$, for all $\lambda \in \mathbb{C}^{*}$. The $\mathbb{P}^{2}$ wrapped by the instanton is located at $z=0$, and the orientifold involution that fixes the $O 7^{-}$plane acts geometrically as

$$
\sigma: s \leftrightarrow-s
$$

Since we are interested in contributions to the superpotential, our task is to show that the instanton only has the two universal zero modes $\theta^{\alpha}$, with every other possible zero mode absent, see [9] for a review of D-brane instanton physics. These zero modes can be either neutral $\bar{\tau}_{\dot{\alpha}}$ modes, coming from the instanton being non-invariant, neutral deformation modes coming from the instanton not being rigid, or charged zero modes coming from massless strings between the instanton and the background D-branes.

\subsection{Neutral zero modes}

The absence of neutral zero modes (i.e., those of deformation and $\bar{\tau}_{\dot{\alpha}}$ types) can be rephrased as the instanton being rigid and of $O(1)$ type, and can be shown as follows. First of all, since $\mathbb{P}^{2}$ is rigid, there are no deformation modes. By explicitly writing the transition functions it is not difficult to see that $\Omega$ is invariant under the orientifold involution (3.1), i.e. it satisfies (2.1). In order to prove that the instanton is of $O(1)$ type we need to show that the gauge symmetry on the instanton in the absence of the orientifold is $U(1)$, and that the orientifold projects this to the $O(1)$ component (as opposed to projecting down to $U S p)$. The fact that the rank 2 bundle preserves a $U(1)$ gauge group can be understood by noting that the structure group of $\mathbb{P}^{2}$, being Kähler, is $U(2)$, while the local symmetry on the rank 2 bundle is also $U(2)$. The preserved gauge group is the commutant of both factors, and it is thus $U(1)$. A more formal but also more systematic way of showing this comes from simply computing $\operatorname{Ext}^{p}\left(i_{*} \Omega, i_{*} \Omega\right)$, which is the space of massless adjoint fermions [10]. For this particular bundle we have $\operatorname{Ext}^{0}\left(i_{*} \Omega, i_{*} \Omega\right)=\mathbb{C}$, and 
by Serre duality on $X$ we also have $\operatorname{Ext}^{3}\left(i_{*} \Omega, i_{*} \Omega\right)=\mathbb{C}$. The Ext groups can be shown to vanish for all other values of $p$. The fact that the projection induced by the orientifold involution is of type $O(1)$ can be seen by a local argument of monodromy around the orientifold locus [11], which shows that the $\bar{\tau}_{\dot{\alpha}}$ modes are the ones projected out. A different way of understanding these last two facts will be given below when we study the instanton at small volume.

\subsection{Charged zero modes}

We are left to show that the charged zero modes - massless strings stretching between the instanton and background D-branes - are absent. In our particular configuration the latter correspond to the $D 7$ branes required to cancel the tadpole induced on the hyperplane of the $\mathbb{P}^{2}$ by the $O 7^{-}$. We perform the zero mode computation at a particular point in the moduli space of the $D 7$ branes where they restrict to a stack of 4 D7 branes wrapping a quadratic curve on $\mathbb{P}^{2}$. If there is a non-vanishing superpotential at this particular point in moduli space, by continuity of the superpotential this shows that generically the superpotential is non-vanishing.

The spectrum of charged zero modes in our particular configuration is counted by [7]:

$$
\operatorname{Ext}^{p}\left(j_{*} \mathcal{F}, i_{*} \Omega\right)=H^{p-1}\left(\mathcal{C},\left.\left.\Omega\right|_{\mathcal{C}} \otimes \mathcal{F}^{\vee}\right|_{\mathcal{C}} \otimes N_{\mathcal{C} \mid \mathbb{P}^{2}}\right)
$$

Here $\mathcal{C}$ denotes the curve (a quadratic $\mathbb{P}^{1} \subset \mathbb{P}^{2}$ ) where the $D 7$ branes and the instanton worldvolume intersect, $N_{\mathcal{C} \mid \mathbb{P}^{2}}$ is the normal bundle of this $\mathbb{P}^{1}$ in $\mathbb{P}^{2}$, given by $\mathcal{O}(4)$ and $\mathcal{F}$ is the bundle of the $D 7$ flavor brane stack, with $j$ the embedding of the D7 stack into $X$. (We will only need the restriction of $j$ to $\mathcal{C}$, given in eq. (3.4) below.) In order to calculate the spectrum (3.2) we thus need to compute the restrictions of the appropriate cotangent bundles to $\mathcal{C}$. Since $\mathcal{C}$ is just a $\mathbb{P}^{1}$, by a well-known result due to Grothendieck we have that $\Omega$, being of rank 2 , splits into a sum of two line bundles when restricted to $\mathcal{C}:\left.\Omega\right|_{\mathcal{C}}=\mathcal{O}(m) \oplus \mathcal{O}(n)$. In addition, the sums of the individual degrees of the line bundles are constrained by the following relation:

$$
n+m=\int_{\mathcal{C}} c_{1}\left(\left.\Omega\right|_{\mathcal{C}}\right)=\int_{\mathbb{P}^{2}} c_{1}(\Omega) \wedge 2 \ell=-6 .
$$

To determine $m$ and $n$ separately we follow the approach in [12]. Let us focus on the local neighborhood of $\mathbb{P}^{2}$, the instanton cycle. We take $\mathcal{C}$, the quadratic curve in $\mathbb{P}^{2}$ wrapped by the $D 7$ branes, to be given by $s^{2}-t u=0$. We can also parameterize $\mathcal{C}$ by the projective coordinates $\left(x_{1}, x_{2}\right)$ with an embedding in $X$ given by

$$
\left(x_{1}, x_{2}\right) \mapsto(s, t, u, w)=\left(x_{1} x_{2}, x_{1}^{2}, x_{2}^{2}, 0\right) .
$$

Let us first compute the restriction $\left.T_{X}\right|_{\mathcal{C}}$ of the tangent bundle of the Calabi-Yau $X$ to $\mathcal{C}$, using the toric Euler sequence restricted to $\mathcal{C}$ :

$$
\left.\left.0 \rightarrow \mathcal{O}_{\mathcal{C}} \stackrel{i}{\rightarrow}\left(\mathcal{O}_{X}(1)^{\oplus 3} \oplus \mathcal{O}_{X}(-3)\right)\right|_{\mathcal{C}} \stackrel{f}{\rightarrow} T_{X}\right|_{\mathcal{C}} \rightarrow 0 .
$$


It is easy to see that $\left.\mathcal{O}_{X}(n)\right|_{\mathcal{C}}=\mathcal{O}_{\mathcal{C}}(2 n)$, for instance by computing the following integral:

$$
\begin{aligned}
k & =\int_{\mathcal{C}} c_{1}\left(\mathcal{O}_{\mathcal{C}}(k)\right)=\int_{\mathcal{C}} c_{1}\left(\left.\mathcal{O}_{X}(n)\right|_{\mathcal{C}}\right) \\
& =\int_{\mathbb{P}^{2}} n \ell \wedge 2 \ell=2 n .
\end{aligned}
$$

The exact sequence to study is thus:

$$
\left.0 \rightarrow \mathcal{O}_{\mathcal{C}} \stackrel{i}{\rightarrow} \mathcal{O}_{\mathcal{C}}(2)^{\oplus 3} \oplus \mathcal{O}_{\mathcal{C}}(-6) \stackrel{f}{\rightarrow} T_{X}\right|_{\mathcal{C}} \rightarrow 0 .
$$

The map $i$ is given by the inclusion of $\mathcal{C}$ into $X$, which acts on the sections as:

$$
i=\left(\begin{array}{c}
x_{1} x_{2} \\
x_{1}^{2} \\
x_{2}^{2} \\
0
\end{array}\right) .
$$

The map $f$ is defined by imposing exactness of (3.7), i.e., $f \circ i={ }^{t}\left(\begin{array}{lll}0 & 0 & 0\end{array}\right)$, and it is thus given by:

$$
f=\left(\begin{array}{cccc}
x_{1} & -x_{2} & 0 & 0 \\
x_{2} & 0 & -x_{1} & 0 \\
0 & 0 & 0 & 1
\end{array}\right)
$$

Imposing that the degrees of the bundles in (3.7) make sense, we thus obtain $\left.T_{X}\right|_{\mathcal{C}}=$ $\mathcal{O}_{\mathcal{C}}(3) \oplus \mathcal{O}_{\mathcal{C}}(3) \oplus \mathcal{O}_{\mathcal{C}}(-6)$.

In order to find the restriction of $T_{\mathcal{S}}$ from the restriction of $T_{X}$ we use the adjunction formula:

$$
\left.\left.\left.0 \rightarrow T_{D}\right|_{\mathcal{C}} \stackrel{K}{\rightarrow} T_{X}\right|_{\mathcal{C}} \stackrel{L}{\rightarrow} \mathcal{O}_{X}(D)\right|_{\mathcal{C}} \rightarrow 0
$$

where $D=\mathbb{P}^{2}$ is the divisor of interest. In our particular case this becomes

$$
\left.\left.0 \rightarrow T_{D}\right|_{\mathcal{C}} \stackrel{K}{\rightarrow} \mathcal{O}_{\mathcal{C}}(3) \oplus \mathcal{O}_{\mathcal{C}}(3) \oplus \mathcal{O}_{\mathcal{C}}(-6) \stackrel{L}{\rightarrow} \mathcal{O}_{X}(D)\right|_{\mathcal{C}} \rightarrow 0
$$

with $D$ given by $w=0$. In general, if $D$ is defined by a homogeneous polynomial equation $P=0$, we have that the map (in the exact sequence before restriction) $\widehat{L}: T_{X} \rightarrow \mathcal{O}(D)$ is given by $\sum p_{i} \frac{\partial}{\partial t_{i}} \rightarrow \sum p_{i} \frac{\partial P}{\partial t_{i}}$, where $t_{i}$ are the toric coordinates $(s, t, u, w)$, and $p_{i}$ a homogeneous polynomial of the same degree as $t_{i}$. Let us perform explicitly the restriction of the tangent bundle to $\mathbb{P}^{2}$. In this case $P=w$. The map $L=\left(\begin{array}{lll}a_{1} & a_{2} & a_{3}\end{array}\right)$ is then given by the solution to:

$$
\left(\begin{array}{lll}
a_{1} & a_{2} & a_{3}
\end{array}\right)\left(\begin{array}{cccc}
s & -t & 0 & 0 \\
t & 0 & -s & 0 \\
0 & 0 & 0 & 1
\end{array}\right)=\left(\begin{array}{llll}
0 & 0 & 0 & 1
\end{array}\right)
$$

Solving these equations, we have $\left(\begin{array}{lll}a_{1} & a_{2} & a_{3}\end{array}\right)=\left(\begin{array}{lll}0 & 0 & 1\end{array}\right)$, which is compatible with the fact that $\left.O_{X}(D)\right|_{\mathcal{C}}=\mathcal{O}_{\mathcal{C}}(-6)$. This tells us that the map $L$ is simply picking the last component 
in the $\mathcal{O}_{\mathcal{C}}(3) \oplus \mathcal{O}_{\mathcal{C}}(3) \oplus \mathcal{O}_{\mathcal{C}}(-6)$ bundle, discarding the rest. From exactness of (3.11), this implies that $\left.T_{\mathbb{P}^{2}}\right|_{\mathcal{C}}=\mathcal{O}_{\mathcal{C}}(3) \oplus \mathcal{O}_{\mathcal{C}}(3)$, or equivalently by dualization $\left.\Omega\right|_{\mathcal{C}}=\mathcal{O}_{\mathcal{C}}(-3) \oplus \mathcal{O}_{\mathcal{C}}(-3)$.

We also need to find the restriction to $\mathcal{C}$ of the bundle $\mathcal{F}$ on the $D 7$ flavor brane stack. The situation here is less well defined, as in principle in order to specify the bundle one needs to consider the whole compact divisor wrapped by the flavor brane. However, at least locally it makes sense to choose a trivial bundle. In particular, the total space $\mathrm{O}$ of the $\mathcal{O}(-3)$ fiber over the quadratic $\mathbb{P}^{1}$ curve $\mathcal{C}$ has the structure of a toric space with coordinates $\left(x_{1}, x_{2}, p\right)$, identified under the rescaling $\left(x_{1}, x_{2}, p\right) \sim\left(\lambda x_{1}, \lambda x_{2}, \lambda^{-6} p\right)$, with $\lambda \in \mathbb{C}^{*}$. The local intersections are given by $x_{1} \cdot p=x_{2} \cdot p=1, p \cdot p=-6$. We identify $p=0$ with $\mathcal{C}$. The canonical class of the space is then given by $K_{\mathrm{O}}=-D_{1}-D_{2}-D_{p}$, and we immediately see that locally the cycle wrapped by the flavor brane is Spin, since the intersection of $K_{\mathrm{O}}$ with the local class $D_{p}$ is even. So, locally we can choose a trivial (physical) flux on the flavor brane. In the conventions used in this paper, where we work in terms of the sheaves defining the D-branes, we have that $\mathcal{F}=\sqrt{K_{\mathrm{O}}}$ (so $\mathcal{F} \otimes \sqrt{K_{\mathrm{O}}^{\mathrm{V}}}$ is trivial). In particular, we have the restriction $\left.\mathcal{F}\right|_{\mathcal{C}}=\sqrt{\left.K_{\mathrm{O}}\right|_{\mathcal{C}}}=\mathcal{O}_{\mathcal{C}}(2)$, since $\left.K_{\mathrm{O}}\right|_{\mathcal{C}}=\mathcal{O}_{\mathcal{C}}(4)$. As one may expect, this choice is also invariant under the orientifold action [8]:

$$
\begin{aligned}
\left.\mathcal{F}\right|_{\mathcal{C}}=\mathcal{O}_{\mathcal{C}}(2) \longrightarrow & \left.\left.\mathcal{F}^{\vee}\right|_{\mathcal{C}} \otimes K_{\mathrm{O}}\right|_{\mathcal{C}} \\
& =\mathcal{O}_{\mathcal{C}}(-2) \otimes \mathcal{O}_{\mathcal{C}}(4)=\mathcal{O}_{\mathcal{C}}(2)
\end{aligned}
$$

where we have again restricted to the curve, in order to not have to involve global information.

Now that we have obtained the restriction of the gauge bundles to the curves, we can easily compute the full spectrum by plugging $\left.\mathcal{F}\right|_{\mathcal{C}}$ and $\left.\Omega\right|_{\mathcal{C}}$ into (3.2):

$$
\begin{aligned}
\operatorname{Ext}^{1}\left(j_{*} \mathcal{F}, i_{*} \mathcal{E}\right) & =H^{0}\left(\mathcal{C}, \mathcal{O}_{\mathcal{C}}(-3)^{\oplus 2} \otimes \mathcal{O}_{\mathcal{C}}(-2) \otimes \mathcal{O}_{\mathcal{C}}(4)\right) \\
& =H^{0}\left(\mathcal{C}, \mathcal{O}_{\mathcal{C}}(-1)^{\oplus 2}\right)=0
\end{aligned}
$$

Similarly, using Serre duality and the fact that $X$ is Calabi-Yau we have that $\operatorname{Ext}^{1}\left(i_{*} \mathcal{E}, j_{*} \mathcal{F}\right)=$ $\operatorname{Ext}^{2}\left(j_{*} \mathcal{F}, i_{*} \mathcal{E}\right)=H^{1}\left(\mathcal{C}, \mathcal{O}_{\mathcal{C}}(-1)^{\oplus 4}\right)=0$. So, there are no charged zero modes, and we see that the instanton does give rise to a non-vanishing superpotential $W_{\text {non-pert }}=$ $A_{1-\text { loop }} e^{-S_{\text {inst }}}$, where $A_{1-\text { loop }}$ is the non-zero one-loop determinant and $S_{\text {inst }}$ is the instanton action. In general we have that $\operatorname{Re}\left(S_{\text {inst }}\right)=\left|Z\left(i_{*} \Omega\right)\right| / g_{s}$, with $Z\left(i_{*} \Omega\right)$ the central charge of the instanton. At large volume this reduces to $\operatorname{Re}\left(S_{\text {inst }}\right)=2 \operatorname{Vol}(D) / g_{s}$, with $\operatorname{Vol}(D)$ the volume of the $\mathbb{P}^{2}$ on which we are wrapping the instanton. Notice in particular the factor of 2 multiplying the volume, coming from the fact that we are considering a rank two stack. So at large volume there is an extra suppression of the non-perturbative effect compared to the case of a hypothetical rank one instanton on the same cycle which - had there been such a contribution to the superpotential — would have gone as $\operatorname{Re}\left(S_{\text {inst }}\right)=\operatorname{Vol}(D) / g_{s}{ }^{1}$ As we go towards small volumes $\alpha^{\prime}$ corrections to the large volume expression for $Z\left(i_{*} \Omega\right)$ become important, and the suppression due to the rank of the bundle is less pronounced. (A couple of particularly interesting points in Kähler moduli space are the quiver point

\footnotetext{
${ }^{1}$ We would like to thank the referee for pointing out a missing factor of two in the expression for the instanton action given in the original version of this paper, and emphasizing the resulting suppression.
} 
analyzed in the next section, in which $Z\left(i_{*} \Omega\right)$ coincides with the central charge for certain rank one branes wrapping the same cycle, and deep in the orbifold phase, where one can even have $Z\left(i_{*} \Omega\right)=0[10]$.)

Note that the instanton contribution to the superpotential may vanish if $\mathcal{F}$ is another vector bundle on the $D 7$ flavor brane stack. For example, a different natural choice is $\mathcal{F}=$ $\Omega_{\mathrm{O}}$, the cotangent bundle for the divisor $\mathrm{O}$, with $\mathcal{F}$ invariant under the orientifold action and the Freed-Witten anomaly cancellation conditions satisfied. It is a simple exercise using the technology described above to show that $\left.\Omega_{\mathrm{O}}\right|_{\mathcal{C}}=\mathcal{O}_{\mathcal{C}}(-2) \oplus \mathcal{O}_{\mathcal{C}}(6)$. Computing the zero modes in this case we obtain:

$$
\begin{aligned}
\operatorname{Ext}^{1}\left(j_{*} \mathcal{F}, i_{*} \mathcal{E}\right)= & H^{0}\left(\mathcal{C}, \mathcal{O}_{\mathcal{C}}(-3)^{\oplus 2} \otimes\right. \\
& \left.\left(\mathcal{O}_{\mathcal{C}}(2) \oplus \mathcal{O}_{\mathcal{C}}(-6)\right) \otimes \mathcal{O}_{\mathcal{C}}(4)\right) \\
= & H^{0}\left(\mathcal{C}, \mathcal{O}_{\mathcal{C}}(3)^{\oplus 2} \oplus \mathcal{O}_{\mathcal{C}}(-5)^{\oplus 2}\right) \\
= & \mathbb{C}^{8}
\end{aligned}
$$

and similarly $\operatorname{Ext}^{1}\left(i_{*} \mathcal{E}, j_{*} \mathcal{F}\right)=\mathbb{C}^{8}$. Thus, there are eight pairs of vector-like zero modes, and the contribution to the superpotential vanishes. There is no contradiction between this result and the one above, since both bundles live in different components of the moduli space. In particular, the two bundles induce different amounts of D3 charge, and thus are connected by emission/absorption of mobile $D 3$ branes. Thus, this provides partial information about the dependence of the instanton action on the moduli of (mobile) D3 branes. In particular, it shows the existence of zeros in the one-loop determinant of the instanton contribution to the superpotential when the D3 branes hit the instanton [13].

\section{Small volume interpretation}

With the contribution of the instanton described by $i_{*} \Omega$ to the superpotential established, let us present an alternative viewpoint that arises when we take the configuration to the quiver point. Specifically, let the volume of the $\mathbb{P}^{2}$ be zero, which turns the smooth space $X$ into the well-known $\mathbb{C}^{3} / \mathbb{Z}_{3}$ orbifold.

We also momentarily forget about the instanton, and instead consider $2 N$ regular $D 3$ branes probing the singularity of the $\mathbb{C}^{3} / \mathbb{Z}_{3}$ orbifold, in addition to the $O 7^{-} / D 7$ stack. Since we are setting $B=0$ this is the quiver point for the $D 3$ branes [10], and the $D 3$ branes decompose into fractional branes as they hit the singularity. The resulting spectrum

\begin{tabular}{|c|c|c|c|}
\hline & $U S p(2 N)$ & $U(2 N)$ & $\mid[U(4)]$ \\
\hline$X^{i} \quad(i \in 1,2,3)$ & $\square$ & $\bar{\square}$ & 1 \\
\hline$A^{j} \quad(j \in 1,2)$ & 1 & $\theta$ & 1 \\
\hline$S$ & 1 & $\square$ & 1 \\
\hline$Q$ & 1 & $\square$ & 4 \\
\hline
\end{tabular}
is obtained using CFT methods, or more generally dimer model methods [14]. One obtains a theory with $U S p(2 N) \times U(2 N)$ gauge group and $U(4)$ flavor group, with the following matter content: 
The same result can also be derived using large volume language. The three elementary fractional branes can be described by the objects $i_{*} \mathcal{O}(-1)[0], i_{*} \Omega[1]$ and $i_{*} \mathcal{O}(-2)[2]$. (Here the numbers in square brackets denote the position of the given sheaf in the associated sheaf complex describing the brane [10], and will not be essential in what follows.) The main point is that the orientifold action (3.1) leaves the $i_{*} \Omega[1]$ brane invariant, leading to the $\operatorname{USp}(2 N)$ factor, while it exchanges $i_{*} \mathcal{O}(-1)[0]$ and $i_{*} \mathcal{O}(-2)[2]$, giving rise to the $U(2 N)$ factor (once we take $2 N$ such objects). The matter content can be determined by computing Ext groups between the fractional branes, and the $U(4)$ global symmetry factor comes from the $D 7$ brane stack wrapped on the quadratic divisor.

The most important part of this discussion for our purposes is the gauge group $U S p(2 N)$ associated to the invariant node. Gauge instantons for $\operatorname{USp}(2 N)$ field theories have gauge symmetry $O(1)$. In particular, the euclidean $D 3$ brane describing the field theory instanton will be of type $O(1)$, i.e., given by the same sheaf as the field theory brane. Since the USp stack is associated with $i_{*} \Omega$ the gauge instanton in this node is precisely the instanton we have been studying. In fact, it is not necessary to have $N \neq 0$. If $N=0$ the invariant node gives a $U S p(0)$ "gauge group", which also gives rise to non-perturbative string theory dynamics [15-17], due to $O(1)$ D-brane instantons [18].

\section{Conclusions}

We have shown the existence of an $O(1)$ type instanton $i_{*} \Omega$, invariant under the orientifold involution, in the particular case of the complex Calabi-Yau cone over $\mathbb{P}^{2}$. Although the discussion focused on a particular example, it is clear that the analysis holds for essentially any rigid cycle, and that the basic phenomenon will be ubiquitous in type IIB string compactifications.

A natural extension of this work would be to consider higher rank instantons, ideally classifying all bundles satisfying (2.1). Furthermore, even rigidity is not a necessary condition for instanton contributions when worldvolume fluxes are taken into account [19]. It would be interesting to study the general conditions under which non-rigid, non-Spin cycles contribute to the superpotential once one considers all solutions to (2.1).

Finally, we expect the non-perturbative effects discussed in here to play an important role in the stabilization of Kähler moduli in type IIB compactifications. As an example, in [20] we show how the $O(1)$ instanton discussed in this paper can be used to stabilize the Kähler moduli in a model introduced in [21].

\section{Acknowledgments}

We thank A. Collinucci, R. Donagi, R. Savelli and A. Uranga for illuminating discussions. P.B. thanks the hospitality of the Simons Center for Geometry, the Niels Bohr Institute, and the theory groups at CERN and DESY, where much of this work was carried out. I.G.-E. would like to thank N. Hasegawa for kind encouragement and support. The work of P.B. is supported by NSF grants PHY-0645686 and PHY-1207895. 
Open Access. This article is distributed under the terms of the Creative Commons Attribution License which permits any use, distribution and reproduction in any medium, provided the original author(s) and source are credited.

\section{References}

[1] F. Denef, M.R. Douglas and B. Florea, Building a better racetrack, JHEP 06 (2004) 034 [hep-th/0404257] [INSPIRE].

[2] V. Balasubramanian, P. Berglund, J.P. Conlon and F. Quevedo, Systematics of moduli stabilisation in Calabi-Yau flux compactifications, JHEP 03 (2005) 007 [hep-th/0502058] [INSPIRE].

[3] D.S. Freed and E. Witten, Anomalies in string theory with D-branes, Asian J. Math 3 (1999) 819 [hep-th/9907189] [INSPIRE].

[4] R. Blumenhagen, X. Gao, T. Rahn and P. Shukla, A Note on Poly-Instanton Effects in Type IIB Orientifolds on Calabi-Yau Threefolds, JHEP 06 (2012) 162 [arXiv:1205.2485] [INSPIRE].

[5] M. Cicoli, S. Krippendorf, C. Mayrhofer, F. Quevedo and R. Valandro, D-Branes at del Pezzo Singularities: Global Embedding and Moduli Stabilisation, JHEP 09 (2012) 019 [arXiv: 1206.5237] [INSPIRE].

[6] E. Sharpe, Lectures on D-branes and sheaves, hep-th/0307245 [INSPIRE].

[7] S.H. Katz and E. Sharpe, D-branes, open string vertex operators and Ext groups, Adv. Theor. Math. Phys. 6 (2003) 979 [hep-th/0208104] [INSPIRE].

[8] D.-E. Diaconescu, A. Garcia-Raboso, R.L. Karp and K. Sinha, D-Brane Superpotentials in Calabi-Yau Orientifolds, Adv. Theor. Math. Phys. 11 (2007) 471 [hep-th/0606180] [INSPIRE].

[9] R. Blumenhagen, M. Cvetič, S. Kachru and T. Weigand, D-Brane Instantons in Type II Orientifolds, Ann. Rev. Nucl. Part. Sci. 59 (2009) 269 [arXiv:0902.3251] [inSPIRE].

[10] P.S. Aspinwall, D-branes on Calabi-Yau manifolds, hep-th/0403166 [INSPIRE].

[11] M. Cvetič, I. Garcia-Etxebarria and R. Richter, Branes and instantons at angles and the F-theory lift of O(1) instantons, AIP Conf. Proc. 1200 (2010) 246 [arXiv:0911.0012] [INSPIRE].

[12] S. Katz, On the finiteness of rational curves on quintic threefolds, Comp. Math. 60 (1986) 151.

[13] O.J. Ganor, A Note on zeros of superpotentials in F-theory, Nucl. Phys. B 499 (1997) 55 [hep-th/9612077] [INSPIRE].

[14] S. Franco, A. Hanany, D. Krefl, J. Park, A.M. Uranga, et al., Dimers and orientifolds, JHEP 09 (2007) 075 [arXiv: 0707.0298] [inSPIRE].

[15] M. Aganagic, K.A. Intriligator, C. Vafa and N.P. Warner, The Glueball superpotential, Adv. Theor. Math. Phys. 7 (2004) 1045 [hep-th/0304271] [INSPIRE].

[16] K.A. Intriligator, P. Kraus, A.V. Ryzhov, M. Shigemori and C. Vafa, On low rank classical groups in string theory, gauge theory and matrix models, Nucl. Phys. B 682 (2004) 45 [hep-th/0311181] [INSPIRE]. 
[17] M. Aganagic, C. Beem and S. Kachru, Geometric transitions and dynamical SUSY breaking, Nucl. Phys. B 796 (2008) 1 [arXiv:0709.4277] [InSPIRE].

[18] I. Garcia-Etxebarria, D-brane instantons and matrix models, JHEP 07 (2009) 017 [arXiv:0810.1482] [INSPIRE].

[19] M. Bianchi, A. Collinucci and L. Martucci, Magnetized E3-brane instantons in F-theory, JHEP 12 (2011) 045 [arXiv:1107.3732] [INSPIRE].

[20] P. Berglund and I. García-Etxebarria, Kähler Moduli Stabilization in Calabi-Yau Manifolds with Branes at Toric Singularities, to appear.

[21] V. Balasubramanian, P. Berglund, V. Braun and I. Garcia-Etxebarria, Global embeddings for branes at toric singularities, JHEP 10 (2012) 132 [arXiv:1201.5379] [INSPIRE]. 